\title{
Historia de las ideas filosóficas: un método del filosofar latinoamericano*
}

\author{
[Artículos]
}

\author{
Fecha de entrega: 28 de mayo de 2020 \\ Fecha de evaluación: 20 de septiembre de 2020 \\ Fecha de aprobación: 15 de noviembre de 2020
}

Nicolás Tamayo Leal ${ }^{* *}$

Citar como:

Tamayo, N. (2021). Historia de las ideas filosóficas: un método del filosofar latinoamericano. Cuadernos de Filosofía Latinoamericana, 42(124).

https://doi.org/10.15332/25005375.5852

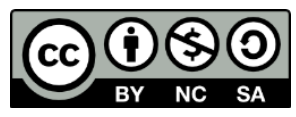

\section{Resumen}

En este artículo se exponen, previa presentación de algunas

consideraciones iniciales sobre el pensamiento filosófico

latinoamericano, las características y fundamentos principales de la

historia de las ideas filosóficas latinoamericanas como un marco

adecuado para pensar nuestros problemas propios. A propósito de esta

disciplina filosófica, su justificación y su vigencia, se presentan algunas

críticas hechas a esta tradición de pensamiento desde la filosofía

\footnotetext{
* Este artículo es producto del trabajo de grado presentado por el autor para optar al título de abogado en la Universidad Colegio Mayor de Cundinamarca.

** Abogado de la Universidad Colegio Mayor de Cundinamarca. Correo:

nicolastm.70@gmail.com; ORCID: https://orcid.org/0000-0002-1745-0011
} 
posmoderna y contemporánea, las cuales han puesto de presente entre nosotros algunos pensadores colombianos. Culmina con la consideración de esta historiografía como un método filosófico "nuestroamericano".

Palabras clave: filosofía latinoamericana, historia de las ideas, identidad, normalización.

\section{History of philosophical ideas: a method of Latin American philosophizing}

\section{Abstract}

This article presents, after some initial considerations on Latin American philosophical thought, the main characteristics and foundations of the history of Latin American philosophical ideas as a suitable framework for thinking about our own problems. Regarding this philosophical discipline, its justification and its validity, some criticisms made to this tradition of thought from postmodern and contemporary philosophy are presented, which have been brought to our attention by some Colombian thinkers. It concludes with the consideration of this historiography as an "our American" philosophical method.

Keywords: Latin American philosophy, history of ideas, identity, normalization.

Vamos a estudiar la filosofía evidentemente: pero a fin de que este estudio, por lo común tan estéril, nos traiga alguna ventaja positiva, vamos a estudiar, como hemos dicho, no la filosofía en sí, no la filosofía aplicada a la teoría abstracta de las ciencias humanas, sino la filosofía aplicada a los

objetos de un interés más inmediato para nosotros; en una palabra, la

Cuadernos de Filosofía Latinoamericana

ISSN: 0120-8462 | e-ISSN: 2500-5375 | DOI: https://doi.org/10.15332/25005375

Vol. 42 N.o 124 | enero-junio de 2021 
filosofía política, la filosofía de nuestra industria y riqueza, la

filosofía de nuestra literatura, la filosofía de nuestra religión y nuestra historia. Decimos de nuestra política, de nuestra industria, en fin, de todas aquellas cosas que son nuestras, porque lo que precisamente forma el carácter y el interés de la enseñanza que ofrecemos es que ella se aplica a investigar la razón de conducta y de progreso de estas cosas entre nosotros.

JUAN BAUTISTA ALBERDI, Ideas para un curso de filosofía contemporánea

A fuerza de andar buscando los comienzos se convierte uno en un cangrejo. El historiador mira hacia atrás; al final cree también hacía atrás.

FRIEDRICH NIETZSCHE, Crepúsculo de los ídolos

\section{Introducción}

En el primer apartado, el escrito indica cómo la discusión acerca del universalismo, como pretensión de toda filosofía, condujo poco a poco al surgimiento de un variado pensamiento latinoamericano que ha pensado al continente en clave de su liberación. Dentro del espectro amplio de disciplinas que componen ese pensamiento, se reconoce a la historia de las 
ideas latinoamericana como una disciplina más que ha conducido hacia ese fin.

La segunda parte del texto precisa que esa historia de las ideas ha tenido un desarrollo histórico con diversos hitos a lo largo de los siglos XIX y XX. En ese devenir, ha tenido renovaciones metodológicas internas que han ampliado su alcance, así como han contribuido a que mejore sus resultados al ser parte de los esfuerzos de los pueblos del continente por conocerse. Se presenta una definición de esa historia de las ideas latinoamericana como una disciplina filosófica no neutral, encargada de indagar por el funcionamiento, el valor y la influencia social de las ideas a través de un análisis dialéctico entre la idea y el contexto de su surgimiento.

Luego, se enuncia que esta forma particular de hacer filosofía, con todo y su relativa indefinición epistemológica y metodológica, por sus apuestas políticas y la importancia que ha tenido para el desarrollo mismo del pensamiento filosófico en la región, hace parte de la forma como América Latina se ha entendido y como se ha expresado en un contexto histórico particular. Razón por la cual es vigente para dar cuenta de las ideas y su función social, así como de los contextos en los que surgieron, con el fin de evidenciar los efectos que pudieron o no tener en la región. Aunque para tal efecto sea necesario incluir los aportes y discusiones sobre los enfoques metodológicos mencionados por las críticas. Finalmente, se concluye que la historia de las ideas, en el continente, ha fungido como un camino muy importante para el desarrollo de la actividad filosófica en América Latina y el Caribe.

En términos metodológicos, el diseño que se usó para la elaboración de este artículo obedece a las siguientes consideraciones. En primer lugar, se 
trató de una investigación de corte teóricoํ. Debido al interés perseguido (Habermas, 1973), se ubicó en el paradigma crítico-hermenéutico, en tanto que se abordan un conjunto de concepciones sobre el mundo guiadas por la interpretación crítica de los textos y contextos, que tienen pretensiones de transformación. En razón al tipo de datos tratados y a la manera como el autor entiende la realidad social, así como para efectos de delimitar el objeto de análisis, el enfoque de este trabajo fue el cualitativo (BonillaCastro y Rodríguez, 2005). Se hizo uso de la metodología documental, en cuanto que se leyeron, analizaron y comentaron textos clásicos y contemporáneos sobre la historia de las ideas latinoamericanas, sus antecedentes y críticas. Las técnicas de las que se valió el autor fueron la exploración temática, el rodeo teórico y el análisis cualitativo y descriptivo.

\section{De la filosofía práctica al pensamiento latinoamericano}

Es ya un lugar común decir que fue el argentino Juan Bautista Alberdi quien, en el año de 1842 mientras se encontraba exiliado en Montevideo, por vez primera se refirió a la necesidad de hablar de una filosofía (nuestro)americana. Lo hizo en el texto Ideas para un curso de filosofía contemporánea, en el cual planteó que no existía una filosofía universal, ya que no hay una solución universal a las cuestiones que traza el pensamiento filosófico, pues esas soluciones dependen de cada sujeto, época y nación (Alberdi, 1978, p. 6). Con esto quedaba abierta la puerta para proponer una filosofía en estas tierras como se había hecho en otras.

La propuesta de Alberdi en ese texto es la de una filosofía práctica que hace uso de lo más selecto del pensamiento europeo para ser aplicado a

\footnotetext{
${ }^{1}$ En todo caso, no debe entenderse por ese simple hecho que lo aquí presentado carezca de efectos prácticos. Está claro que práctica y teoría son dos caras de un mismo proceso que se complementan en tanto etapas (sin preeminencia una sobre la otra) y que se suceden en el tiempo: lo teorizado debe ser practicado y esto debe ser reflexionado a la luz de la teoría.
}

Cuadernos de Filosofía Latinoamericana

ISSN: 0120-8462 | e-ISSN: 2500-5375 | DOI: https://doi.org/10.15332/25005375

Vol. 42 N. 0124 | enero-junio de 2021 
"nuestra industria y riqueza, $[\ldots]$ nuestra literatura, [...] nuestra religión y nuestra historia" pues él consideraba que "América practica lo que piensa [...] Europa" (Alberdi, 1978, pp. 10-11). Por ende, debían ponerse en funcionamiento esas teorías, en especial aquellas de filosofía política, para solucionar las problemáticas americanas que surgían con posterioridad a las independencias políticas.

Sin embargo, este argentino no solo se refutará más adelante, sino que también planteará el argumento que posteriormente le permitirá a la generación de la tradicionalmente llamada "normalización filosófica" superar su propuesta y profundizar la pregunta por una filosofía propiamente latinoamericana. Él dirá que la filosofía americana debía nacer de las necesidades de nuestras naciones y sería filosofía en tanto que "[resolviera] el problema de los destinos americanos" (Alberdi, 1978, p. 12).

Así, filosofía se haría en todas partes y no solo en Europa y su particularidad serían los problemas a los que debe plantar cara en cada tiempo y lugar. De acuerdo con el colombiano Leonardo Tovar (2009), la filosofía práctica propuesta en este texto por Alberdi generaría una ruptura con la filosofía especulativa de Europa y vendría a ser la "carta fundacional del latinoamericanismo filosófico" (p. 258).

De lo anterior se debe destacar, entonces, que se habla de filosofía latinoamericana porque se trata de un pensamiento situado que no elude ni encubre desde dónde y para quién se teoriza. Esto en la medida que su pretensión está relacionada, al menos en un principio, con la búsqueda de una respuesta a la pregunta por la "identidad" de estos pueblos y se encuentra estrechamente vinculada con su nacionalidad, desde donde se piensan problemas propios como los de la liberación política y cultural. 
Como refiere Tovar (2009): "la capacidad de pensar nuestra realidad en cada momento de modo auténtico, reflexivo y crítico ha sido y será lo que garantice la posibilidad y la existencia de un filosofar latinoamericano" (p. 261). Así pues, no se dice latinoamericana por chauvinismo o porque no aspire a dialogar con los planteamientos filosóficos de otras latitudes. Antes bien, como lo ha sostenido Dussel (1996) desde su filosofía analéctica de la liberación, su propósito también es comenzar por vez primera el verdadero proceso de mundialización de la filosofía, antes propiedad exclusiva del Mediterráneo, al buscar un diálogo filosófico global (p. 10).

Por eso, esta filosofía latinoamericana no se preocupa hoy de la sistemática crítica que se le hiciera desde el centro del sistema-mundo, con eco en las élites criollas de la periferia tan acostumbradas al colonialismo intelectual, acerca de su ausencia de universalidad o de su excesivo localismo. Acertadamente, esta filosofía ha sabido adoptar lo que para la ciencia social propuso Orlando Fals Borda (2014), esto es, que el rigor es lo que hace universal, o no, a un determinado planteamiento filosófico o científico, sin importar el lugar desde el cual se produce (p. 74). Igualmente, tal como lo ha mencionado Horacio Cerutti (2011), esta filosofía latinoamericana, después del debate ${ }^{2}$ suscitado en la mitad del siglo XX entre el peruano Augusto Salazar Bondy y el mexicano Leopoldo Zea, no precisa continuar discutiendo alrededor de si se trata de una filosofía de o en Latinoamérica; y mucho menos es dable mantener la crítica a este tipo de pensamiento como "folklor o aldeanismo" (falta de universalidad a la que se aludió anteriormente), ni se sostienen los

\footnotetext{
2 El cual se puede revisar en los textos de Salazar Bondy (1996) y Zea (1998). Para una lectura sugerente de dicha polémica como punto de origen para el planteamiento de una filosofía de la liberación, se puede revisar el texto del profesor Mario Ruíz (2020).
} 
cuestionamientos acerca de una supuesta ausencia de rigor o erudición (p. 82).

Pero es cierto que no hay una sola filosofía latinoamericana, sino filosofías y otro tipo de saberes que se han construido desde América Latina y el Caribe. Los cuales han configurado una cierta unidad problemática 3 , a pesar de haber surgido en un ambiente similar, así como en el marco de la proliferación, a mediados del siglo pasado, de varios movimientos de liberación nacional y de perseguir, fundamentalmente, los mismos objetivos 4 .

De acuerdo con Cerutti (2011), hacen parte de estas filosofías de o para la liberación latinoamericana, cuatro corrientes: la ontologicista, representada inicialmente por Rodolfo Kusch y Mario Casalla; la analéctica de Enrique Dussel, quizá la más difundida alrededor del globo; la historicista, de Arturo Andrés Roig; y la problematizadora, del mismo Cerutti, Severino Croatto y Manuel Ignacio Santos (p. 86)5.

Así, el pensamiento latinoamericano surgió como una forma de autoconocimiento y autoafirmación de estos pueblos. De ahí que teorías económicas, sociológicas, pedagógicas y teológicas también hayan estado

\footnotetext{
3 Trasladando al campo de la filosofía lo que ha planteado José Aricó (1999) a propósito de la difusión del marxismo en Latinoamérica. Esta unidad problemática se configura en tanto que las filosofías nacionales responden a aspectos similares, pero con sus condicionamientos e intereses particulares: a pesar de hablar, por ejemplo para el caso de Iberoamérica, una misma lengua y de tener un pasado (¿y presente?) de saqueo y explotación común, o de haberse independizado relativamente al mismo tiempo, cada nación, en todo caso, presenta unas particularidades que se articulan con esas generalidades continentales.

${ }^{4}$ Esto es: "impulsar una reflexión de fuerte impronta cultural, cargando el énfasis en las dimensiones de la identidad y de la búsqueda de la integración de la región" (Cerutti, 2011, p. 79).

${ }^{5}$ Sin embargo, no conviene adentrarnos acá en profundizar sobre esa taxonomía. Sirva la clasificación del filósofo argentino no más para indicar la variedad de manifestaciones filosóficas que se pueden enmarcar en lo latinoamericano, dentro de las cuáles interesa tener presente la corriente historicista, cuyo énfasis está puesto en la disciplina de la historia de las ideas como parte del método del filosofar en esta región.
} 
en una constante relación de discusión permanente y de mutua influencia con ese pensamiento filosófico, debido a lo cual hacen parte de esos esfuerzos por los pueblos de la América Latina de indagar por su identidad histórica.

En ese orden de ideas, vale la pena mencionar, brevemente, otras formas del pensamiento nuestroamericano que han influenciado a esa filosofía y, por supuesto, a la historia de las ideas filosóficas en la región, tales como: las teorías de la dependencia y su férrea oposición a las tesis desarrollistas junto con su aportación teórica de la fundamental distinción entre el centro y la periferia del moderno sistema económico mundial (Jaguaribe et al., 2017); la pedagogía liberadora del maestro brasilero Paulo Freire (2012; 2013) que tanto ha inspirado variados movimientos y prácticas educativas populares en América Latina y el mundo, a la par que ha transformado la forma misma de la enseñanza y, en especial, de la alfabetización; así como la teología de la liberación, sus aportes a la organización campesina y popular desde los ejercicios de las Comunidades Eclesiales de Base y cuyo hito puede encontrarse en la Conferencia General del Episcopado Latinoamericano llevada a cabo en 1968 en la ciudad de Medellín (muy poco tiempo después de la muerte del colombiano Camilo Torres Restrepo, uno de los curas cuya práctica más influyó en esa propuesta); finalmente, no se podría dejar de mencionar la sociología de la liberación, militante, comprometida y popular que siguiendo a Camilo Torres, teorizó Orlando Fals Borda (2013) y con la cual ha legado a Latinoamérica la investigación acción participativa, una original propuesta de estudio sobre los órdenes sociales y una brillante apuesta revolucionaria denominada socialismo raizal.

Por todo lo anterior, en este artículo se adopta una perspectiva, de entre todo ese conjunto de saberes latinoamericanos, llamada historia de las 
ideas filosóficas latinoamericana, dejando claro que dicha disciplina no agota todo ese rico pensamiento ni la filosofía nuestroamericana.

\section{Historia e ideas en nuestra américa}

¿Qué es la tan mencionada historia de las ideas? Como varios aspectos de la filosofía misma, una respuesta a esa pregunta va a depender de las fuentes en que se sustente. Aun así, puede decirse que hay un cierto consenso en América Latina acerca de qué se puede entender por esta disciplina, incluso cuando existen múltiples trabajos, metodologías y apuestas que puedan distanciarse en algunos elementos y que aciertan en señalar una relativa indefinición de esta. Previo a presentar una definición, acá se expone un pequeño recuento de su constitución histórica y se precisan elementos característicos desde los cuales se aborda su estudio en este escrito.

De acuerdo con el filósofo colombiano Damián Pachón Soto (2015), siguiendo a José Luís Abellán, cuatro teorías de la filosofía del español Ortega y Gasset son indispensables para la constitución de la historia de las ideas en América Latina. Al menos en la versión inicial de José Gaos y Leopoldo Zea antes de los años cuarenta del siglo pasado. Estas son las de la circunstancia, las generaciones, las ideas y las creencias, de las cuales es importante resaltar la primera, pues es la que lleva a proponer una "historia de las ideas como de las circunstancias", en tanto que la circunstancia humana es siempre histórica y toda idea tiene siempre una circunstancia (2015, p. 16).

No obstante, previo a toda esa influencia orteguiana, ya se puede observar un incipiente germen de esa historia de las ideas en los trabajos de algunos pensadores latinoamericanos del siglo XIX como José Enrique Rodó, José Toribio Medina y Juan María Gutiérrez; aunque es cierto que esos 
esfuerzos se dieron en el ámbito de la crítica literaria, pero con una suerte de "actitud valorativa" hacia los trabajos de los iberoamericanos. Específicamente en el ámbito filosófico (que será muy importante para el desarrollo posterior de esta forma de historiar las ideas en el continente), serán unas polémicas de ese siglo propuestas por algunos filósofos, las que pondrán de presente "la función social de la idea, respecto de nuestra realidad americana" y fungirán como fuente de trabajos posteriores propiamente de historia de las ideas filosóficas (Roig, 2013, p. 180). Sin embargo, es en la época de la llamada "normalización filosófica"6, esto es, alrededor de 1940, cuando se constituye e institucionaliza la historia de las ideas y empieza a tener una difusión e importancia inesperadas. Esa importancia tiene que ver, en principio, con que "surgió y se desarrolló como una de las tantas formas culturales de autoafirmación de nuestros pueblos" fruto de lo cual, nos dice Roig, "ha superado hace tiempo el mero interés erudito que pueda haberla movido en alguna ocasión, para convertirse en una imprescindible herramienta de autoconocimiento [continental]" (2013, pp. 178-179). Herramienta que se vería impulsada por las demandas de liberación nacional que han ido presentándose en la región desde la mitad del siglo pasado y en adelante.

Un desarrollo de la historia de esta forma de hacer filosofía en la región, desde esa época normalizadora hasta finales del siglo pasado e inicios de este, puede ser planteado, muy resumidamente, en estos términos, atendiendo a la periodización sugerida por Cerutti (1997). Dos corrientes de esta forma de hacer historia en la región aparecen en la década del

\footnotetext{
${ }^{6}$ Es decir, cuando se da "el nacimiento de la filosofía como quehacer específico, con un lugar institucional dentro de nuestras universidades" (Roig, 2013, págs. 181-182) o, en otras palabras, cuando se profesionaliza la filosofía y deja de ser un campo de extraños y esporádicos interesados que se encuentran situados fuera de la academia, para pasar a ser una actividad formativa reconocida institucionalmente. Para una crítica de esta categoría puede revisarse el texto de Betancur (2015) y en clave de crítica historiográfica el de Bernal (2020).
} 
cuarenta del siglo pasado, la de Francisco Romero (impulsor de la idea de la normalización filosófica) y la de José Gaos (desde la circunstancia orteguiana). La de este último fue la que más desarrollo tuvo. En este periodo surgen revistas, instituciones y publicaciones que demostraban la fecundidad de esa propuesta. Posteriormente, hacia los cincuenta y sesenta, de la mano de la teoría de la dependencia, el panameño Ricaurte Soler propuso articular ambas perspectivas latinoamericanas con el fin de evitar el idealismo adjudicado al historicismo y la causalidad externalista criticada a las teorías de la dependencia; hecho que contribuyó a clarificar políticamente a la historia de las ideas. Sobre la década del setenta, fue Roig quien impulsó lo que se denominó "ampliación o renovación metodológica”, con lo cual se debía incluir, en los ejercicios historiográficos latinoamericanos, los aportes de la semiótica y la teoría de las ideologías. Desde entonces, en la región se crean programas, instituciones, centros de pensamiento, revistas, congresos, bibliotecas, proyectos conjuntos, etc., que dan cuenta del avance de la disciplina, su discusión con el pensamiento posmoderno y la importancia que ha cobrado en América Latina y el mundo, así como se pueden observar aún hoy cultivadores de ese tipo particular de hacer filosofía (Cerutti y Magallón, 2003, pp. 38-42).

Visto someramente su origen y desarrollo histórico, acá una definición preliminar de lo que puede entenderse por la historia de las ideas filosóficas: esta vendría a ser el ejercicio teórico que indaga acerca de la función y el valor de las ideas y su "inserción social y nacional" (Roig, 2013, p. 205) con pretensión de transformación hacia el futuro. Así, este ejercicio historiográfico no se consuela con la mera presentación de autores, sus obras y sus ideas en una sucesión cronológica de su aparición por mero interés letrado o académico. Por el contrario, este tipo de historia exige reconstruir críticamente el contexto social y político de producción 
de esas ideas, así como la influencia que las mismas tuvieron en su medio. Es decir, interesa más la función social de las ideas que las ideas por sí mismas.

La anterior definición, sin embargo, plantea una cierta ambigüedad acerca de lo que debería entenderse por la idea y su función social, por lo cual es necesario aclarar su alcance. No se asume que las ideas tengan el poder por sí mismas de determinar el desarrollo social, político, cultural o económico de una nación, un sector social o un individuo. Eso sería propio de una concepción idealista que desconocería a todas luces la materialidad de la existencia humana, con lo cual, invirtiendo las palabras de Marx (2008), se aceptaría que es la conciencia (ideas) la que determina al ser social y no al revés, como lo sostuvo acertadamente el filósofo de Tréveris (p. 5).

No obstante, es cierto que las ideas sí influyen y condicionan (aunque no determinan inexorablemente) nuestro conocimiento de lo que existe y, por ende, su transformación, toda vez que esas ideas median la relación entre los individuos y el mundo. De igual forma, las lecturas sobre la realidad que puede tener una persona van a estar permeadas por aspectos como su pertenencia, o no, a un determinado género, etnia, clase o grupo social dentro de las relaciones sociales y de producción en una época específica. Lo que se reconoce con esto, entonces, es que hay una mutua influencia o condicionamiento entre las ideas y las circunstancias materiales de existencia de los sujetos que las expresan, siendo ese hecho lo que interesa a esta historia cuando se pregunta por la función social de una idea.

Toda vez que América Latina no ha sido la única región en el globo que ha dedicado esfuerzos entorno a la historia de las ideas, se deben mencionar algunas características específicas que tiene esta disciplina entre nosotros, como lo son: su búsqueda por la concreción de una filosofía 
latinoamericana; las referidas intenciones de autoafirmación en clave de identidad cultural, nacional y continental; y el problema que ha generado a estos pueblos el asunto de la dependencia política, económica y cultural (Roig, 2005, p. 532).

Sumado a lo anterior, en términos políticos, debe enunciarse que esta disciplina no es neutralmente valorativa. Por medio de las importantes investigaciones acerca del pensamiento nuestroamericano, así como de su inserción e influencia en el mundo social, los constructores de esta historiografía han apoyado la "lucha contra la dependencia externa, el imperialismo y el colonialismo [...así como han] reivindicado la autonomía de América Latina. Por estas razones, también [han] fomentado la construcción de realidades alternativas propias, es decir, utopías" como la de la integración latinoamericana (Pachón Soto, 2015, pp. 18-19). Como puede advertirse, este quehacer filosófico no es ajeno a la realidad social vigente. Por el contrario, impulsa su transformación aportando elementos desde el estudio crítico de la tradición de pensamiento latinoamericana. Es necesario dar paso ahora a una presentación acerca de cuestiones metodológicas, con el fin de identificar los elementos indispensables a tener en cuenta para adelantar este ejercicio historiográfico.

En primer lugar, es importante señalar que la reflexión metodológica realizada por los autores latinoamericanos en el marco de esta disciplina, se ha llevado a cabo con la intencionalidad de politizar el método y las cuestiones epistemológicas, antes que reflexionar por el método en sí mismo (Pachón Soto, 2015, p. 19). Esto en atención al carácter marcadamente político que ha tenido esta forma de filosofar y que ha sido un lugar común en otros saberes latinoamericanos, como se enunció antes. De esa manera, también debe quedar claro que lo metodológico no es presentado acá como una receta, unos pasos a seguir o una ruta muy bien 
definida que señala cómo y qué se debe hacer para lograr una historia de las ideas. Antes bien, se presentan cuestiones teóricas y recomendaciones que sirven de horizonte y reflexión permanente en esta labor.

En ese sentido, en el capítulo "Algunas pautas sobre el pensamiento latinoamericano", Arturo Andrés Roig (2013) presentó una serie de máximas a tener en cuenta cuando se estudia, historiza y produce el pensamiento nuestroamericano. En sus palabras "estas siete pautas [...] tienen la pretensión de develar el fundamento de posibilidad del pensar latinoamericano y el modo cómo hemos de llegar a pensar latinoamericanamente" (2013, pp. 108-109). Las normas presentadas por él son:

- $\quad$ Hacer filosofía como filosofía sin más.

- Tenernos como valiosos para nosotros mismos.

- Tomar conciencia de los límites del "nosotros mismos".

- Asumir lúcidamente nuestra radical historicidad.

- Determinar y denunciar las formas de alienación de nuestro pensamiento.

- Tomar conciencia de la unidad de destino en las sucesivas etapas de realización del "nosotros mismos".

- Reelaborar los campos del saber de nosotros mismos, como formas de un saber de liberación. (2013, p. 108)

En resumen, estas pautas nos presentan una serie de elementos de importante consideración al valorar el pensamiento latinoamericano estudiado. Por ejemplo, hacer filosofía sin más es entender que toda filosofía es universal, en tanto que sea fruto de la acción de pensar los aspectos de la vida cotidiana, material, realmente existente. Sin embargo, ese pensar, esa acción que es el filosofar, no escapa del tiempo y el lugar que le corresponde, lo cual dota de especificidad y particularidad sus 
planteamientos en tanto y en cuanto los reconoce y los hace explícitos de manera crítica.

Igualmente, en una estela hegeliana, Roig (2013) ha dicho sobre el "tenernos como valiosos a nosotros mismos" que esta máxima en el pensamiento americano tiene que ver con la necesidad de evidenciar la constitución de una conciencia sobre el mundo, una conciencia de sí y, fundamentalmente, una conciencia para sí que evoca al sujeto latinoamericano como valioso para el pensamiento y, por ende, busca mejorar sus condiciones de vida (2013, pp. 105-122).

En todo caso, ese ser valiosos se presenta en tanto existencia humana y no por el hecho de ser latinoamericano. Lo que se desea es el reconocimiento que debemos realizar sobre nosotros una vez se ha tomado conciencia de la alienación en la que nos encontramos y fruto de lo cual también debemos identificar los límites de esa valoración con otros pueblos, así como reconocer su historicidad y no darlo por sentado en un análisis a priori.

Con todo y la profundidad de las pautas presentadas, aún cabe la posibilidad de ser más específicos en cuanto al método (entendido como camino que puede seguirse de muy variadas formas). A ese propósito se organizó en México, en septiembre de 1974 y convocada por la Unesco, una reunión de expertos sobre la historia de las ideas en América Latina, en cuya comisión II $^{7}$ se elaboraron unas recomendaciones metodológicas, las cuales citamos in extenso debido a su importancia y concreción:

\footnotetext{
7 De esta comisión hicieron parte Leopoldo Zea (México), Francisco Miró Quesada (Perú), Arturo Andrés Roig (Argentina), Arturo Ardao (Uruguay), Guillermo Francovich (Bolivia), Abelardo Villegas (México), Ramón Xirau (México), Roberto Fernández Retamar (Cuba), José Antonio Portuondo (Cuba), Elías Pino Iturrieta (Venezuela), Luis Navarro de Brito (Brasil), Javier Ocampo López (Colombia) y María Elena Rodríguez Ozán (Argentina).
} 
- Se recomienda partir de una concepción de la idea entendida como un elemento significativo que integra una estructura más amplia, con todas las connotaciones de este último término (económicas, políticas, etc.), dando cabida además a las ideas en sus diversas manifestaciones: filosofemas, vivencias, ideologías, concepciones del mundo, etc.

- Se recomienda aplicar un tratamiento dialéctico a la historia de las ideas, subrayando principalmente dos aspectos: la conveniencia de encararla desde nuestro presente, y la necesidad de señalar a la vez los condicionamientos sociales y el poder transformador de la idea.

- Se recomienda no abordar la historia de las ideas como historia académica, abriéndose a la incorporación de las ideologías y en particular a las de los grandes movimientos de liberación e integración latinoamericanos, frente a las ideologías de dominación.

- Se recomienda encarar la historia de las ideas no a partir de campos epistemológicos (filosofía, política, etc.), sino de problemas concretos latinoamericanos y las respuestas dadas a cada uno de ellos desde aquellos campos.

- $\quad$ Se recomienda tratar todo desarrollo de historia de las ideas latinoamericanas a partir del supuesto de la unidad del proceso histórico de Latinoamérica.

- $\quad$ Se recomienda ir más allá de una historia de las ideas de tipo nacional y avanzar hacia uno más amplio de regiones continentales, sin olvidar el supuesto señalado antes.

- Se recomienda señalar en lo posible la función de las influencias en relación con los procesos históricos propios.

- Se recomienda dar preferencia a la historia de las ideas entendida como historia de la conciencia social latinoamericana. (Moreno Fraginals y Lafaye, 1975, p. 5) 
Adicional a las anteriores recomendaciones, pautas metodológicas y epistemológicas, Cerutti (1997) nos previene sobre una serie de problemas que es importante tener presente al abordar este tipo de historia.

En primer lugar, lo relativo a la necesidad de cuestionar la relación entre idea y realidad en la afirmación "pensar la realidad". En efecto, no se puede dejar pasar de manera inadvertida que ese pensar la realidad, que se busca encontrar en autores y sus obras, no escapa de cuestionamientos teóricos sobre el conocimiento y el acceso por medio de este a la realidad. Es acá donde cobra una especial relevancia incluir, en términos metodológicos, los análisis del lenguaje y las ideologías que, precisamente, permiten identificar en buena medida la influencia que tienen aquellos y sus manifestaciones en la configuración y acceso a la realidad estudiada y representada por las ideas de los autores bajo análisis (Cerutti, 1997, pp. 189-194).

Otro tanto sucede con la relación entre filosofía e historia de la filosofía. Es indispensable entender que en América Latina no se ha adelantado una historia de la filosofía desde la perspectiva de la historia de las ideas, sino que esta disciplina ha permitido cuestionar constantemente la existencia o no de una filosofía latinoamericana, a la vez que ha ido demostrando, con su ejercicio, la posibilidad de existencia de ese filosofar nuestroamericano. Así, no se trataría de periodizar, seleccionar obras y autores en forma cronológica y señalar sus ideas, sino de discutir permanentemente si es eso filosofía latinoamericana, para lo cual es importante estudiar sus planteamientos en perspectiva histórica, atendiendo a los contextos y relaciones internas y externas con esa filosofía (Cerutti, 1997, pp. 194202). 


\section{Críticas a la historia de las ideas latinoamericana}

Luego de presentar lo que podríamos llamar algunos hitos y periodos importantes en el desarrollo histórico de la historia de las ideas latinoamericana, así como clarificada la definición o la manera como en este trabajo se entiende esa disciplina filosófica y expuestas algunas recomendaciones metodológicas para adelantar ese ejercicio, es propicio pasar a estudiar algunas de las más recientes críticas que se le han hecho, con el fin de justificar la vigencia de la misma para historiar el pensamiento en la región.

Uno de los trabajos que más ha cuestionado a la historia de las ideas y, por supuesto, a la filosofía latinoamericana en general, es el del colombiano Santiago Castro-Gómez (1999), con su ya clásico texto Crítica de la razón latinoamericana, en especial en su capítulo cuatro, quien reconoce en la introducción que esas críticas tienen antecedentes en los filósofos colombianos Daniel Herrera Restrepo y Roberto Salazar Ramos (pp. 9-14).

Su principal cuestionamiento a este tipo de filosofía tiene que ver con que al articularse desde el lenguaje y presupuestos modernos para atacar el humanismo europeo que se impuso universalmente, el humanismo latinoamericano estaría impregnado de los mismos problemas que presenta ese humanismo que critica. En otras palabras, la preocupación fundamental en la crítica del profesor Santiago es que la historia de las ideas buscaría encontrar algo "auténtico o propio" en la filosofía latinoamericana, con lo cual cabría la posibilidad que se revivieran esencialismos con tendencia hacia los chovinismos y los totalitarismos ${ }^{8}$.

\footnotetext{
${ }^{8}$ Esta crítica surge del contexto nihilista de "pérdida de sentido del mundo, de la vida y de la historia que sería y sigue siendo un slogan de la filosofía posmoderna" (Pachón Soto, 2013, p. 29). Es decir, en el contexto de toda esa crítica a las promesas de la razón, el iluminismo, la religión, el marxismo y el pensamiento social crítico. En pocas palabras: la crisis de la modernidad, que, entre otras cosas, resulta de la vivencia de
} 
De ahí que él señale la existencia de una aparente "razón histórica", fundamento de esos esencialismos, que estaría presente en los planteamientos básicos tanto de la filosofía latinoamericana como de la historia de las ideas. Y que iría desde Ortega hasta Roig, pasando por Gaos y Zea. Se trataría, entonces, de esa filosofía de la historia, ese metarelato de la razón histórica, que tanto ha insistido en rechazar el pensamiento posmoderno y poscolonial, que fungen como marco teórico del colombiano.

Esa razón histórica se evidenciaría, según Castro-Gómez, en cada uno de esta manera: en Ortega se puede encontrar en su consideración que las ideas o creencias fundamentales en una sociedad (producidas por las élites intelectuales en una misión salvífica de la circunstancia actual y fruto de una lectura del pasado para evitar errar en el futuro) son las que impulsan los cambios históricos que las producen; a su vez, en Gaos, quien habría latinoamericanizado la filosofía orteguiana, estaría en el planteamiento sobre la necesidad de revisar, retrospectivamente, el desarrollo de las ideas en el continente, con el fin de ver de cómo los pensadores habrían dado cuenta de su circunstancia. Con lo cual se evidenciaría el interés de esta filosofía, tributaria de la tradición inmanentista de la modernidad, por las dinámicas de conformación de los estados nacionales y de la identidad cultural (Castro-Gómez, 1999, pp. 100-104).

Por su parte, en Zea, ese circunstancialismo se encontraría presente en su obra a través de la filosofía de la historia de Hegel, que le permitiría presentar la asunción penosa del pensamiento americano en una sucesión de etapas (que irían desde la colonia hasta mediados del siglo pasado). La cual le permitiría al continente no solo salvar su propia circunstancia, sino

\footnotetext{
catástrofes sociales del siglo XX como las grandes guerras o los totalitarismos (impulsados, precisamente, por esencialismos religiosos, nacionalistas, ideológicos, etc.).
} 
la circunstancia universal como consecuencia de la proyección de un nuevo humanismo que surgiría de esa historicidad y que no universalizaría un humanismo particularizado (Castro-Gómez, 1999, pp. 105-109).

Finalmente, en Roig, indica que su propuesta de historia de las ideas y, por tanto, de filosofía de la historia, se compondría de tres cosas básicas: 1) mencionar los momentos históricos en los que "se han dado procesos de autoconstitución de un 'sujeto latinoamericano", para 2) evaluar lo hecho por ese pensamiento en esos procesos, con el fin de 3) encontrar las utopías “decantadas en la tradición filosófica latinoamericana que pudieran servir como 'ideales regulativos' para orientar la historia del continente según fines racionales"(Castro-Gómez, 1999, pp. 111). Estos planteamientos del argentino se fundamentarían, según Castro-Gómez (1999), en dos ideas: la del imperativo moral kantiano que debe seguir la historia, pero atendiendo a un sujeto empírico que se reconoce como valioso y como fin en sí mismo; y en la tesis hegeliana del amo y el esclavo. De la unión de ambas ideas se tendría, entonces, un sujeto colectivo que iría adquiriendo autoconsciencia a lo largo de su despliegue histórico, reconociendo los aspectos de la dominación en la que se encuentra para identificar los caminos de su liberación.

De esa lectura hecha sobre la filosofía latinoamericana se derivaría que el ser humano es el sujeto por excelencia que hace la historia y cuyas ideas generan los cambios históricos. Por lo cual, no quedaría más que confiar en las élites letradas para adelantar los cambios que requiere la sociedad para alcanzar su telos. Siguiendo a Ángel Rama, Castro-Gómez (1999) pretende demostrar que lo que ha inspirado la realización de la filosofía latinoamericana ha sido el deseo de los letrados e intelectuales por ordenar y diciplinar a la sociedad. De lo cual no se podría suponer ningún ideal acerca de un fin noble o de elevación del latinoamericano, sino solo de cierto tipo de latinoamericano, cuya consecuencia evidente es que se cae 
en la misma dinámica del humanismo europeo y conduce, o estaría en potencia de hacerlo, hacía distintos totalitarismos (Castro-Gómez, 1999, pp. 113-120).

Otro colombiano que también ha puesto de presente algunas críticas que se le han hecho a esta disciplina es el filósofo Leonardo Tovar González (2017), quien lo hizo en su artículo "La historia de las ideas en América Latina, modelo para desarmar".

En ese escrito, Tovar expone la historia de las ideas latinoamericana como un enfoque eminentemente filosófico9. El programa investigativo de ese enfoque estaría dirigido a identificar la repercusión histórica de las ideas en punto de sus efectos materiales y simbólicos. Teniendo presente que esa repercusión "hace parte de la historia misma de la verdad de esas propuestas filosóficas, de manera que el análisis de la validez" (Tovar, 2017, p. 46) de cualquier doctrina filosófica no se terminaría con el estudio y confrontación de sus tesis frente a otras escuelas, sino que requeriría tener presente sus efectos históricos y filosóficos en el contexto de su nacimiento, o en el de otros sitios. Con esa intención, en clara diferencia con Castro-Gómez, no importa que ese análisis se haga desde la genealogía foucaultiana o desde las influencias literarias, pues es un aspecto de método que no afectaría la intención investigativa.

Así, tres consecuencias habría que considerar al concebir a la historia de las ideas como una suerte de historia de la filosofía latinoamericana. Inicialmente 1) presentaría una teleología histórica, aunque no sea la hegeliana, pues considera que la verdad se desarrolla a través del tiempo;

\footnotetext{
9 Para ese fin, no obstante señalar la hegeliana división entre historia de la filosofía (encargada de la búsqueda de la verdad a través de las teorías filosóficas) y la historia de las ideas (cuyo fin es indagar por la influencia de las ideas en la sociedad sin atención a su validez), se refiere a la historia de las ideas latinoamericana como un ejercicio filosófico en sí mismo que no tendría nada que ver con esa distinción.
}

Cuadernos de Filosofía Latinoamericana

ISSN: 0120-8462 | e-ISSN: 2500-5375 | DOI: https://doi.org/10.15332/25005375

Vol. 42 N.o 124 | enero-junio de 2021 
2) esa búsqueda de la verdad filosófica e histórica precisa de una confianza en el sentido teleológico; y 3) se presenta como la historia de la filosofía latinoamericana misma, en tanto que es ella un ejercicio filosofante latinoamericano que descubre esa filosofía al llevar a cabo el ejercicio historiográfico (Tovar, 2017, pp. 47-48).

Sin embargo, en clave crítica, Tovar (2017) presenta las insuficiencias que comportaría esta disciplina si se le compara con la historia intelectual y la historia de los conceptos que se ha adelantado en el marco de la filosofía política (o con la genealogía). En estas, lo principal no es dar cuenta de las ideas desde una perspectiva del presente que prefigura el sentido de aquellas al encasillarlas en una teleología predefinida por quien investiga, sino dar cuenta de los usos argumentativos de las ideas y de la significación real que adquirieron en su contexto. Para lo cual se debe partir de identificar esos aspectos sociales en el texto mismo, así como de los efectivos usos del lenguaje en el momento que se expresaron y aparecieron esas ideas. De ahí que lo que indagan es el sentido histórico que buscaron comunicar las ideas en cada contexto particular (Tovar, 2017, pp. 50-53).

No obstante lo anterior y como ya se dijo, este filósofo colombiano, en oposición a la crítica hecha por Santiago Castro-Gómez, propone que, aun siendo acertadas las insuficiencias señaladas anteriormente y requiriendo esta historia de las ideas para su reconstrucción la inclusión de los enfoques metodológicos referidos, es innegable que sigue siendo vigente en tanto "programa de investigación dirigido a elaborar una historia de la filosofía que al constituirse en filosofía de la historia latinoamericana, le dé contenido histórico a la búsqueda de una filosofía peculiarmente latinoamericana” (Tovar, 2017, p. 55). 
Además de lo enunciado por Tovar, es pertinente recordar lo que Damián Pachón (2015) ${ }^{10}$ ha dicho sobre la obra de Castro-Gómez, para entender la vigencia que conserva la historia de las ideas y la misma filosofía latinoamericana, sin olvidar las precisiones echas antes.

En primer lugar, el profesor Castro-Gómez reduciría a la filosofía de la liberación, en su versión más difundida, a todo el pensamiento latinoamericano que, como se enunció anteriormente, presenta una gran variedad de disciplinas y planteamientos, incluso contradictorios entre sí, que no es reducible a esa sola corriente filosófica de ese pensamiento (Pachón, 2015, pág. 33). En relación con este punto, además se habría omitido que la historia de las ideas comporta un cierto eclecticismo, una relativa indefinición, que debió ser tenida en cuenta por el referido filósofo en su análisis, por lo cual no la debería reducir a los planteamientos de dos de sus exponentes (Zea y Roig) y dejar de lado la variedad de autores que, desde diversas perspectivas, la han abordado.

En segundo lugar, en cuanto al humanismo que el profesor Gómez-Castro critica en los latinoamericanos estudiados en su texto, se presentarían al menos otros tres reduccionismos:1) indicar que el humanismo existente es solo el moderno; 2) presentar al humanismo latinoamericano como un discurso que se quiere imponer a la especie humana en su conjunto; 3) y exponer la lucha anticolonial como el deseo de ingresar a la modernidad o de desarrollar ese humanismo moderno de Europa en América Latina (Pachón, 2015, pp. 38-39).

\footnotetext{
10 Muy en vía de la crítica de Horacio Cerutti sobre Castro-Gómez: "La intención aparecía como válida, pero sus logros fueron escasos. La argumentación apareció como insuficiente y el soporte historiográfico no resistió la crítica. Las confusiones entre propuestas diversas que aparecen en su texto como unificadas, etc., no permitió cumplir con los objetivos" (Cerutti, 2011, p. 121).
} 
Esos reduccionismos no hacen más que desconocer toda una tradición de pensamiento, incluso europeo, que ha indicado la existencia de varios humanismos. O la posibilidad de discutir y configurar el humanismo como fenómeno histórico y contingente de acuerdo a los movimientos sociales, grupos políticos, sectores y facciones de clase que hay en un espacio y tiempo determinados. Igualmente, conduce a olvidar que ese humanismo latinoamericano ha contribuido a la inclusión en la esfera pública los debates sobre el desarrollo económico, político y social de estos pueblos desde visiones no eurocéntricas o modernas (ibid).

En tercer lugar, frente a Castro-Gómez puede decirse que su crítica a esta disciplina obvia el papel que esta ha jugado en la desvelación de la función social de las ideas y de los intelectuales en la constitución histórica (que no teleológica) del continente. Mediante aquella se ha puesto de presente la responsabilidad de las élites en la configuración de prácticas racistas, se ha denunciado el colonialismo, se ha promovido la integración latinoamericana y se ha estudiado la difusión, circulación y producción de las ideas. Lo cual permite señalar que no es posible aceptar la superación de esta disciplina, así sin más, como él pretende (Pachón, 2015, pp. 40-41). Finalmente, al partir de la consideración que las ideas están mediadas por el lenguaje y que este, a su vez, lo está por las ideologías ${ }^{11}$, se podría despojar a esta disciplina del idealismo que le adjudica el profesor CastroGómez. Motivo por el cual propone la superación o configuración de esa historia de las ideas desde la perspectiva foucaultiana y en el marco de una genealogía de las prácticas. Tesis que debería ser más bien aplicada en el sentido de articular ambas perspectivas, tal como lo proponen Tovar (2017) y el mismo Pachón (2015).

\footnotetext{
${ }^{11}$ Superación del circunstancialismo orteguiano que se dio en la etapa de la ampliación metodológica expuesta en el apartado dos.
}

Cuadernos de Filosofía Latinoamericana ISSN: 0120-8462 | e-ISSN: 2500-5375 | DOI: https://doi.org/10.15332/25005375 Vol. 42 N. ${ }^{\circ} 124$ | enero-junio de 2021 
Con todo, es cierto que el proyecto de la historia de las ideas no puede caer en una valoración bondadosa a priori de lo latinoamericano, situación que en la actualidad no parece encontrar ningún exponente, pero de la que supo advertir y poner de presente Castro-Gómez y que debe ser tenido en cuenta a la hora de elaborar este ejercicio historiográfico.

\section{Corolario}

Filosóficamente hablando, la historia de las ideas, tal y como fue presentada, no es una historia de la filosofía ni una filosofía de la historia.

Se trata más bien de una forma particular de hacer filosofía que ha caracterizado a buena parte de los trabajos de los filósofos latinoamericanos ${ }^{12} \mathrm{y}$, por lo tanto, a la filosofía latinoamericana misma (Roig, 2005, p. 534). Lo que la caracteriza como tal es que la pregunta por la existencia de un pensar enraizado en esta región ha conducido a la edificación de ese mismo pensar latinoamericano.

En un primer momento, se ha filosofado pensando sobre la condición de posibilidad del pensamiento filosófico entre nosotros. Ese método ha permitido hacer importantes descubrimientos. Por ejemplo, sobre el papel de la filosofía en la región para mantener la dominación o propiciar la liberación. Lo que sucede es que la historia de las ideas, al hacer la pregunta por la existencia de la filosofía nuestroamericana, pone en duda cánones, patrones y formas tradicionales (norteurocéntricas) de hacer

\footnotetext{
12 Nótese, como se mencionó antes, que de muy diversas formas se ha hecho filosofía en Latinoamérica y no exclusivamente desde la historia de las ideas, aun cuando puede afirmarse que sí ha sido una de las más recurrentes. En ese sentido es que Pachón Soto (2015) menciona a la filosofía intercultural de Raúl Fornet-Betancourt, la filosofía analéctica de la liberación de Enrique Dussel o el vitalismo cósmico de Darío Botero Uribe como expresiones filosóficas latinoamericanas que no se han remitido a la historia de las ideas para su desarrollo (p. 18).
} 
filosofía, con lo cual se abre el espectro valorativo hacia otros caminos del filosofar que hay en la región.

De esa manera, se hacen otras preguntas que no van dirigidas a buscar lo propio (como se le suele criticar), sino que cuestionan precisamente filosofías de otras latitudes. Opera un reconocimiento del valor intrínseco y de la posibilidad del filosofar en el continente, que genera objeciones a aquellos planteamientos que reducen nuestra condición a meros receptores de lo ya pensado. En ese sentido, esa historia nos permite encontrarnos como negados y nos conmina a negar esa negación, produciendo un cambio cualitativo en nuestra forma de habitar el mundo.

También nos ha permitido valorar con otra perspectiva la producción intelectual nuestroamericana. La tradición filosófica que es estudiada por esa historia confirma que esta funge como un método del filosofar latinoamericano. Esto es así porque aquella tradición es leída en su contexto y no aisladamente. De esa manera, se evidencia que, así como otros pueblos han pensado y resuelto con rigor sus problemas en las diversas dimensiones de la vida, asimismo nosotros hemos ejercido una praxis histórica en esa dirección, que era fuertemente negada e invisibilizada hasta antes de que se adelantara ese ejercicio historiográfico. Luego, ese reconocimiento no solo evidencia que hay filosofía en América del Sur. Además, moviliza otras preguntas que ejercitan el pensar al indagar por los motivos, razones y formas de esa invisibilización. Con todo, se puede afirmar que, aunque no es el único, la historia de las ideas ha sido un método por excelencia para ejercitar el pensamiento filosófico en estas tierras.

\section{Referencias}

Alberdi, J. B. (1978). Ideas para un curso de filosofía contemporánea. Universidad Nacional Autónoma de México. http://ru.ffyl.unam.mx/handle/10391/2953 
Aricó, J. (1999). La hipótesis de justo: escritos sobre el socialismo en América Latina. Sudamericana.

Bernal, L. (2020). Descolonizar la historia de la tradición filosófica en Colombia: la necesidad de reevaluar los postulados historiográficos actuales. Cuadernos de Filosofía Latinoamericana, 41(123), 149-165. https://doi.org/10.15332/25005375/4497

Betancur, J. C. (2015). Para un análisis crítico del concepto de normalización filosófica. Universitas Philosophica, 32(65), 137-157. https://www.redalyc.org/articulo.oa?id=4095/40954.3046006

Bonilla-Castro, E., y Rodríguez, P. (2005). Más allá del dilema de los métodos. La investigación en ciencias sociales. Norma.

Castro-Gómez, S. (1999). Crítica de la razón latinoamericana. Puvill Libros.

Cerutti, H. (1997). Hacia una metodología de la historia de las ideas (filosóficas) en América Latina. Grupo Editorial Miguel Ángel Porrúa.

Cerutti, H. (2011). Doscientos años de pensamiento filosófico nuestroamericano. Ediciones desde abajo.

Cerutti, H., y Magallón, M. (2003). Historia de las ideas latinoamericanas ¿Disciplina fenecida? Casa Juan Pablos.

Dussel, E. (1996). Filosofía de la liberación. Nueva América.

Fals Borda, O. (2013). Socialismo raizal y el ordenamiento territorial. Ediciones desde abajo.

Fals Borda, O. (2014). Ciencia, compromiso y cambio social. Editorial El Colectivo.

Freire, P. (2012). Pedagogía del oprimido. Siglo Veintiuno Editores.

Freire, P. (2013). La educación como práctica de la libertad. Siglo Veintiuno Editores.

Habermas, J. (1973). Conocimiento e interés. Ideas y Valores, 42-45, 61-76. https://revistas.unal.edu.co/index.php/idval/article/view/29111

Jaguaribe, H., Ferrer, A., Wionczek, M. y Santos, T. (2017). La dependencia políticoeconómica en América Latina. Clacso.

Marx, K. (2008). Contribución a la crítica de la economía política. Siglo Veintiuno Editores. 
Moreno Fraginals, M., y Lafaye, J. (1975). Reunión de expertos sobre la historia de las ideas en América Latina y para planificación del nuevo programa sobre las culturas indígenas e inmigratorias. Unesco. https://unesdoc.unesco.org/ark:/48223/pfoooo011839?posInSet=4\&queryId=NEXPLORE-dd293193-66dd-495a-a95e-416cb5eb38b5

Pachón Soto, D. (2013). Preludios filosóficos a "otro mundo posible". Ediciones desde abajo.

Pachón Soto, D. (2015). Estudios sobre el pensamiento filosófico latinoamericano. Ediciones desde abajo.

Roig, A. A. (2005). Historia de las ideas. En R. Salas Astrain, Pensamiento crítico latinoamericano: conceptos fundamentales (Vol. II, pp. 531-550). Ediciones Universidad Católica Silva Henríquez.

Roig, A. A. (2013). Arturo Andrés Roig: metodología y filosofía del pensamiento latinoamericano. Academia Nacional de Historia.

Ruíz, M. (2020). La polémica Salazar Bondy - Leopoldo Zea: de la filosofía de la dominación a la filosofía de la liberación. Erasmus, 22, 238-307. https://www.icala.org.ar/erasmus/Archivo/2020/erasmus\%2022-22020/erasmus-22-2-2020-07-RuizSotelo.pdf

Salazar Bondy, A. (1996). ¿Existe una filosofía de nuestra América? Siglo Veintiuno Editores.

Tovar González, L. (2009). Excurso. Las fundaciones de la filosofía latinoamericana. En E. Dussel, E. Mendieta, y C. Bohórquez, El pensamiento filosófico latinoamericano, del Caribe y “latino" [130o-20oo] (pp. 255-261). Siglo Veintiuno Editores.

Tovar González, L. (2017). La historia de las ideas en América Latina, modelo para desarmar. En G. I. Reyes Corredor y L. Tovar González, Investigaciones en filosofía y cultura en Colombia y América Latina (pp. 41-58). Universidad Santo Tomás.

Zea, L. (1998). La filosofía americana como filosofía sin más. Siglo Veintiuno Editores. 